\title{
Actores en las decisiones públicas: aportes desde el enfoque de análisis de políticas ${ }^{1}$ Actors in public decisions: contributions from the policy analysis approach
}

DOI: http://dx.doi.org/10.17981/econcuc.38.2.2017.04

Recibido: may 30/2017 Devuelto para revisión: jun29/17 Aceptado: sep 12/2017

Jennifer Fuenmayor ${ }^{2}$

\section{Resumen}

Este artículo identifica los actores que intervienen en el proceso de políticas públicas considerando las corrientes teóricas dominantes del enfoque de análisis de políticas. La investigación es no experimental bibliográfica, con un estudio descriptivo. Dentro de este enfoque se escogieron: el ciclo de la política pública de Jones; la configuración de actores y el referencial de política pública de Muller; el marco de las coaliciones promotoras de Sabatier y JenkinsSmith y el modelo decisional de Dente y Subirats. Un aporte importante del enfoque de análisis de políticas es que identifica a los actores que intervienen en las decisiones públicas.

Palabras clave: enfoque de análisis de políticas; decisiones públicas; actores; ciclo de política; el marco de las coaliciones promotoras; enfoque cognitivo.

\begin{abstract}
This article identifies the actors involved in the public policies process, regarding the dominant theories of the policies analysis approach. This is a non-experimental bibliographical research, with a descriptive study. From this approach there were chosen: Jones's public policy cycle; the configuration of actors and the reference of public policy of Muller; the framework of the promoter coalitions of Sabatier \& Jenkins-Smith; and the decisional model of Dente and Subirats. An important contribution of the policy analysis approach is that it identifies the actors that intervene in public decisions.
\end{abstract}

Keywords: policy analysis approach; public decisions; actors; policy cycle; the framework of promoting coalitions; cognitive approach.

\footnotetext{
${ }^{1}$ Este artículo es un producto de investigación del Posdoctorado en Gerencia y Políticas Públicas de la Universidad del Zulia.

${ }^{2}$ Economista. Doctora en Ciencia Política. MSc en Ciencia Política. MSc en Economía. Profesora de la Facultad de Ciencias Económicas y Sociales adscrita al Instituto de Investigaciones Económicas Dionisio Carruyo de la Universidad del Zulia. Miembro de la Academia de Ciencias Económicas del Estado Zulia. Cursante del Postdoctorado en Gerencia y Políticas Públicas (LUZ). Maracaibo, Venezuela. Correo: jennifer.fuenmayor@yahoo.com.ve
} 


\section{Introducción}

Encontrar nuevas herramientas teóricas y métodos de análisis de políticas públicas que estén a tono con la realidad que vivimos en América Latina es una necesidad, considerando tanto la complejidad como los actores involucrados en los procesos políticos de esta región. El objetivo del presente artículo es identificar los tipos de actores que intervienen en el proceso de políticas públicas en las diferentes corrientes teóricas dominantes del enfoque de análisis de políticas públicas.

Se entiende por actor o actores en políticas públicas, un individuo, función, grupo, comité, equipo burocrático, una coalición o incluso el Estado, que busca interferir o moldear una política pública. Asimismo, los actores que participan dentro de una dinámica de una política en juego, se van reconociendo por las posturas de aceptación o rechazo ante una política determinada (Melstner, citado por Santibáñez, Barra y Ortiz, 2005).

Subirats (2015) coincide con Fontaine (2015) en que es en el Estado donde colectivamente hemos de movernos para encontrar salida a problemas que nos afectan a todos, en donde las instituciones públicas son parte necesaria del escenario, del entramado de actores que intervienen en los asuntos propios de las políticas públicas, en otras palabras, de los asuntos que requieren respuestas políticas y colectivas.

En este sentido, es importante mejorar la caja de herramientas para mejorar la práctica de las políticas públicas y, es por eso, que se da la necesidad de realizar aportes en el plano del conocimiento de las políticas públicas. "Analizar las políticas tiene una función científica (mejorar el conocimiento) y política (mejorar la acción y la toma de decisión)" (Fontaine, 2015, p. 6). En el presente artículo nos centramos en la función científica para avanzar en el conocimiento, identificando los tipos de actores que intervienen en cada uno de los modelos seleccionados del enfoque de análisis de políticas, y determinar así si existen aportes considerables de este enfoque para el estudio de los actores.

En referencia al conocimiento en política pública, explica Fontaine (2015) que:

En la actualidad el análisis de políticas públicas en América Latina y el Caribe se enfrenta con varias dificultades. La primera es estructural y es la relativa inmadurez del campo en la región. Fuera de México y Brasil, y en menor medida, Argentina y Colombia, no hay una comunidad epistémica donde se pueda encontrar aportes sustantivos ni lineamientos teóricos de análisis de políticas (...) hay poca producción de conocimiento en la región (...) Las principales editoriales latinoamericanas especializadas tampoco ofrecen mucho material actualizado (...) Entonces hay poca producción y hay poco acceso, en idioma español, a un conocimiento actualizado (...) (p. 7).

De hecho, en América Latina, según Fontaine (2015), no podemos seguir aplicando los mismos conceptos, los mismos métodos, ni siquiera, apoyarnos en los mismos enfoques teóricos tradicionales para entender el proceso político actual.

\section{El enfoque de análisis de políticas en el estudio de las decisiones públicas}

En el enfoque de análisis de la política pública, la pregunta de quién gobierna y cómo gobierna es sustituida por cómo y quién elabora e implementa una política pública (Lagroye, citado por Roth, 2006); es por eso que este enfoque centra su interés hacia la actividad concreta del Estado y, por ende, hacia la importancia que tienen las políticas públicas y cómo éstas determinan la política, las instituciones y el Estado (Roth, 2006).

Este enfoque se centra en el proceso de las políticas públicas, donde "los problemas se conceptualizan y se los presenta al gobierno para que sean solucionados; las instituciones 
gubernamentales formulan alterativas y eligen soluciones de políticas públicas; luego, esas soluciones se implementan, se evalúan y se revisan" (Sabatier, 2010, p. 5).

Una política pública sería el conjunto de acciones y decisiones llevadas a cabo por diversos actores: económicos, sociales y políticos, con la finalidad de resolver un problema definido políticamente como público.

(...) desde el origen norteamericano de la disciplina, el concepto aceptado de la política pública fue el de decisión, que se acompañó con una teoría de decisiones más o menos explícita y sólida. La política pública fue entendida como decisión, como el proceso, contenido y resultado de una decisión del dirigente gubernamental, que define y decide los objetivos, la población objetivo, los medios y recursos, las acciones y los actores responsables (Aguilar, 2017, p. 29).

Los partidarios del enfoque de análisis de políticas afirman que este enfoque permite sacar a la luz a sus actores esenciales (Mény y Thoenig, citados por Roth, 2006). “(...) el análisis de las políticas públicas tiene como objetivo principal la identificación concreta de los actores que intervienen en un proceso de definición, de decisión y de implementación de una política, y asimismo busca poner a la luz las posiciones, intereses y objetivos de esos actores" (Lagroye, citado por Roth, 2006, p. 15).

A juicio de Muller (2006, p. 67),

cuando se busca entender los procesos que conducen a la elaboración y a la puesta en marcha de una política pública, es esencial recordar que una política no es un proceso de decisión abstracto cuyo sentido podría captarse $<<$ desde el exterior $>>$ (...) Es entonces indispensable $<<$ abrir la caja negra $>>$, lo que significa identificar los actores que participan en el policy making para analizar sus estrategias y entender las explicaciones de sus comportamientos.
En referencia a lo anteriormente considerado, dice Muller (2006), que el primer problema a resolver está relacionado con las formas en función de las cuales los actores definen su posición y eligen una $<$ solución $>>$, en concreto se refiere al enfoque de elección racional, el cual proviene de la teoría económica neoclásica y considera que los individuos actúan de acuerdo con sus intereses y que sus preferencias políticas "están determinadas por una lógica que relaciona el costo y los beneficios de las decisiones tomadas" (Muller, 2006, p. 68).

En los procesos de decisiones, los actores no actúan en función de un interés claramente definido que desemboca en una estrategia perfectamente homogénea, tampoco la decisión se explica solamente por la interacción entre una pluralidad de actores, sus preferencias nunca son ni explícitas, ni estables, evolucionan a lo largo del proceso de elaboración y de puesta en marcha de la política (Muller, 2006).

Cabe señalar que el centro de interés de este enfoque en los ochenta estuvo centrado en la problemática de la constitución de las agendas gubernamentales y de las configuraciones (sistemas, redes, comunidades) de actores que influyeron en las políticas públicas (Roth, 2006), tema que vuelve a ocupar un lugar importante en la actualidad cuando se trata de la toma de las decisiones públicas en América Latina.

Finalmente, el enfoque de análisis de las políticas, de acuerdo a Muller (citado por Fontaine, 2015), a nivel epistemológico generó una triple ruptura en la ciencia política en lo que tiene que ver con el estudio del Estado.

En primer lugar, al hacer énfasis en los productos (outputs) y resultados (outcomes), en lugar de los insumos (inputs) (...) el análisis de las políticas públicas rompió con una concepción del Estado como forma de dominación y le sustituyó una concepción centrada en la aptitud de este último a resolver problemas. 
La segunda ruptura puso en duda el carácter racional de la acción pública. Las decisiones no obedecen a una mera racionalidad instrumental que llevaría a la elaboración científica y la ejecución imparcial de las políticas. Por lo contrario, el campo del poder que constituye el Estado está atravesado por las tensiones y las relaciones de fuerza entre los actores, esta alterado por el contexto económico internacional y nacional, está sometido a la instrumentalización, a la irracionalidad y la codicia de los unos y los otros. La tercera ruptura consistió en formular nuevas problemáticas, definir nuevos conceptos y elaborar nuevos métodos de análisis para explicar la actividad concreta del Estado y su funcionamiento (...) (Fontaine, 2015, p. 31).

\section{Metodología}

En este estudio teórico, que se encuentra en la dimensión de la investigación cualitativa, se consideró identificar los tipos de actores que intervienen en la toma de las decisiones en el proceso de formación de las políticas públicas; para ello se escogió el enfoque de análisis de políticas. Se trata de un estudio descriptivo y de revisión documental. Se seleccionaron dentro de este enfoque: 1) el ciclo de la política de Jones; 2) la configuración de actores y el referencial de política pública de Muller; 3) el marco de las coaliciones promotoras de Sabatier y Jenkins-Smith; y 4) el modelo decisional de Dente y Subirats. Los estudios documentales y teóricos derivados de la investigación documental, como una de las opciones de la investigación científica, siguiendo a Balestrini (1998, p. 6),

Son aquellos cuyos objetivos sugieren la incorporación de un esquema de investigación, donde la recolección de información se encuentra vinculada a la delimitación de un diseño bibliográfico (...)
En este sentido, el diseño de investigación bibliográfico o documental implica, un proceso de exploración cognoscitiva que determina la selección de procedimientos tecnooperacionales que deben seguirse para el manejo del dato encontrado en las fuentes documentales, que sirven de base, orientan las formulaciones y delimitan las posibilidades para el análisis creador en este tipo de estudio.

Debido a la naturaleza de la estrategia de investigación para los trabajos documentales y los teóricos, se privilegian aquellos métodos de observación que centran su atención hacia el análisis cualitativo. Por consiguiente, estos métodos se orientan hacia la observación y el análisis de la diversidad de las fuentes documentales (Balestrini, 1998).

\section{Resultados}

Los actores en el enfoque del ciclo de política pública. El ciclo de política pública en, según Roth (2006), propone una descomposición de la política pública en una serie de etapas o de secuencias lógicas. Charles O. Jones identificó a los actores, los procesos y los resultados a lo largo de lo que se llamaría: el ciclo de política en su Introduction to the Study of Public Policy (1970) (Fontaine, 2015). Su propuesta había sido ideada anteriormente por Lasswell ${ }^{3}$.

\footnotetext{
3 "El método de Lasswell consistía en dividir el proceso en etapas, para entender mejor lo que ocurre, quién interviene y qué resultados se pueden esperar en cada momento. Contemplaba siete etapas: inteligencia o entendimiento, promoción o discusión, prescripción o toma de decisión, invocación (entendida como una manera de convencer, por ejemplo, al poder legislativo, de adoptar los medios y los recursos necesarios para ejecutar una decisión), aplicación, terminación y apreciación de la decisión..." (Fontaine, 2015, pp. 53-54).
} 
Es así como esta herramienta propuesta por Jones (1970) distingue cinco fases en la vida o el desarrollo de una política pública: identificación de un problema, formulación de soluciones, toma de decisión, implementación y evaluación (...) tiene la ventaja (y la desventaja) de presentar la política pública como una sucesión de secuencias que corresponden a la representación clásica y racional de la política con sus distintos escenarios y actores (...) Primero, en la fase I, una situación es percibida como problemática por los actores políticos y sociales. Idealmente los partidos políticos, los sindicatos y gremios traducen en su lenguaje el problema que perciben a través de la expresión de sus miembros; se solicita entonces una acción pública y se busca que el problema esté inscrito en la agenda del sistema político (agenda setting). En la segunda fase, una vez lograda la inscripción en la agenda guber- namental, la administración trata de elucidar el problema y propone una o varias soluciones al mismo (policy formulation). Tercero, los actores e instituciones autorizados para tomar la decisión (gobierno, parlamento) examinan la o las soluciones existentes y deciden la respuesta. La cuarta fase concierne a la implementación práctica de la decisión (¡o su no implementación!) Por lo general es la administración la encargada de implementar la solución escogida por el decisor; es decir, de traducir la decisión en hechos concretos (implementation). Al final, la respuesta dada al problema y los impactos causados por su implementación son evaluados por los actores sociales y políticos (policy evaluation) y pueden dar pie a un reinicio de todo el ciclo con el fin de reajustar la respuesta (retroacciónfeedback) o suprimir la política (program termination) (Tabla 1) (Roth, 2006 , pp. 49 y 51).

Tabla 1

El marco del ciclo de política: actividades y actores principales.

\begin{tabular}{|c|c|c|c|c|}
\hline Fase I & Fase II & Fase III & Fase IV & Fase V \\
\hline $\begin{array}{l}\text { Identificación e } \\
\text { institucionalización } \\
\text { de un problema }\end{array}$ & $\begin{array}{l}\text { Formulación de } \\
\text { soluciones o acciones }\end{array}$ & Toma de decisión & Implementación & Evaluación \\
\hline $\begin{array}{l}\text { Actividades } \\
\text { Valores; } \\
\text { acontecimientos; } \\
\text { intereses; } \\
\text { demandas y agenda } \\
\text { pública }\end{array}$ & $\begin{array}{l}\text { Actividades } \\
\text { Elaboración y } \\
\text { valoración de } \\
\text { respuestas; selección } \\
\text { de criterios }\end{array}$ & $\begin{array}{l}\text { Actividades } \\
\text { Encontrar } \\
\text { una coalición } \\
\text { mayoritaria; } \\
\text { legitimación. }\end{array}$ & $\begin{array}{l}\text { Actividades } \\
\text { Ejecución; gestión; } \\
\text { efectos concretos }\end{array}$ & $\begin{array}{l}\text { Actividades } \\
\text { Reacciones; } \\
\text { juicio sobre los } \\
\text { efectos; medición; } \\
\text { valoración; } \\
\text { propuestas de } \\
\text { reajuste }\end{array}$ \\
\hline $\begin{array}{l}\text { Actores } \\
\text { Partidos, } \\
\text { movimientos } \\
\text { sociales, gremios, } \\
\text { medios, políticos, } \\
\text { administración } \\
\text { pública, ONG, etc. }\end{array}$ & $\begin{array}{l}\text { Actores } \\
\text { Parlamentos, gremios, } \\
\text { administración } \\
\text { pública, } \\
\text { organizaciones } \\
\text { políticas y sociales, } \\
\text { ONG, etc. }\end{array}$ & $\begin{array}{l}\text { Actores } \\
\text { Parlamentos, } \\
\text { presidente, } \\
\text { ministro, } \\
\text { gobernador, } \\
\text { alcalde, etc. }\end{array}$ & $\begin{array}{l}\text { Actores } \\
\text { Administración } \\
\text { pública, ONG, } \\
\text { empresa privada, } \\
\text { etc. }\end{array}$ & $\begin{array}{l}\text { Actores } \\
\text { Medios, expertos, } \\
\text { administración } \\
\text { pública, } \\
\text { responsables } \\
\text { políticos, } \\
\text { organizaciones } \\
\text { políticas y sociales, } \\
\text { gremios, afectados, } \\
\text { ONG, etc. }\end{array}$ \\
\hline
\end{tabular}

Fuente: Roth (2008) 
Este modelo, a pesar de sus críticas, ha "logrado avances significativos en la comprensión de la acción del Estado gracias a las numerosas investigaciones empíricas que suscitó el enfoque..." (Roth, 2006, p. 51). Asimismo, "el policy cycle sigue siendo una excelente puerta de entrada pedagógica para presentar el análisis de las políticas públicas..." (Roth, 2006, p. 55).

Para Fontaine (2015), el ciclo de las políticas es un instrumento heurístico, en el sentido de que sirve para organizar la literatura, para organizar el estado del arte en las fases del ciclo de Jones.

(...) Se trata ante todo de un instrumento para ayudarnos a pensar, cuya principal virtud es heurística. No se trata de un instrumento aplicable, tal cual, al análisis, ni mucho menos a la toma de decisión.
A la hora de elegir el tema de análisis que nos interesa, es necesario priorizar un momento del proceso, aunque sea para adquirir un conocimiento más preciso de lo que nos interesa (...) (Fontaine, 2015, p. 56).

En relación con lo anterior, dice Fontaine (2015), que "es muy útil distinguir los momentos de una política para analizar con mayor detalle el conjunto de este proceso: qué ocurre, a qué llevó, quién intervino..." (p. 57), es por eso que el ciclo de Jones nos permite identificar el tipo de actores que intervienen en cada fase del proceso de formación de la política pública (formulación, implementación y evaluación), tanto los actores públicos como también los privados.

Tabla 2

Modelos de cómo ingresan los problemas a la agenda pública de acuerdo con los actores según su poder.

\begin{tabular}{|c|c|}
\hline Modelo & Actores que tienen poder \\
\hline $\begin{array}{l}\text { Modelo de } \\
\text { mediación }\end{array}$ & $\begin{array}{l}\text { Son los políticos (como mediadores entre el Estado y la sociedad) los que hacen la primera } \\
\text { selección de los problemas que deben ingresar en la agenda pública (congresistas, diputados } \\
\text { y concejales). }\end{array}$ \\
\hline $\begin{array}{l}\text { Modelo de } \\
\text { mediatización }\end{array}$ & $\begin{array}{l}\text { Son los medios de comunicación quienes presentan la noticia en prensa, radio y televisión } \\
\text { y ejercen presión para que las autoridades actúen sobre un problema (espacio mediático). }\end{array}$ \\
\hline $\begin{array}{l}\text { Modelo de } \\
\text { movilización }\end{array}$ & $\begin{array}{l}\text { La ciudadanía se moviliza para incluir un tema en la agenda pública (paros, huelgas, } \\
\text { marchas, toma de instituciones) ante la crisis de representación y del modelo de mediación. }\end{array}$ \\
\hline $\begin{array}{l}\text { Modelo de } \\
\text { anticipación }\end{array}$ & $\begin{array}{l}\text { Son las autoridades públicas (gobierno) las que deciden actuar sobre un tema que analizan } \\
\text { como problemático. Detectan y diseñan estrategias de prevención o de mitigación. }\end{array}$ \\
\hline $\begin{array}{l}\text { Modelo de } \\
\text { agenda } \\
\text { internacional }\end{array}$ & $\begin{array}{l}\text { Son los actores internacionales, como los organismos multilaterales (BM; FMI), quienes } \\
\text { ingresan temas que vienen del ámbito internacional en la agenda de los gobiernos, ya sea } \\
\text { por exigencias o compromisos suscritos, como la firma de tratados. }\end{array}$ \\
\hline $\begin{array}{l}\text { Modelo de } \\
\text { agenda nacional }\end{array}$ & $\begin{array}{l}\text { Son los departamentos y los municipios, debido a la distribución de competencias, quienes } \\
\text { ingresan problemas en su agenda para desarrollar directrices del gobierno nacional. }\end{array}$ \\
\hline $\begin{array}{l}\text { Modelo de acción } \\
\text { corporativa } \\
\text { silenciosa }\end{array}$ & $\begin{array}{l}\text { Son los grupos organizados privilegiados quienes tienen acceso a la agenda gubernamental. } \\
\text { La discreción es una condición del modelo. Ej. Cuando se programa la compra de } \\
\text { armamento. }\end{array}$ \\
\hline $\begin{array}{l}\text { Modelo de } \\
\text { agencia judicial }\end{array}$ & $\begin{array}{l}\text { Es el poder judicial quien interviene ante la deficiencia del Estado y la vulneración de } \\
\text { derechos fundamentales. }\end{array}$ \\
\hline $\begin{array}{c}\text { Modelo de } \\
\text { oferta pública }\end{array}$ & $\begin{array}{l}\text { Es el poder legislativo quien interviene en el proceso de formación de la política pública. } \\
\text { Los parlamentarios incorporan en la agenda los temas que son discutidos en la legislatura. }\end{array}$ \\
\hline
\end{tabular}

Fuente: elaboración propia con base en Roth (2006) y Cuervo (2007a). 
Otros modelos ${ }^{4}$ en esta perspectiva de análisis son los que nos muestran en la tabla 2 a continuación Roth (2006) y Cuervo (2007a), en donde se identifica el tipo de actor según su poder para ingresar los problemas a la agenda pública.

Un cierto número de autores, partidarios del enfoque de análisis de políticas, parten en sus investigaciones del enfoque del ciclo de políticas, que permite estudiar concretamente la elaboración de la agenda y la toma de las decisiones públicas.

En relación con lo anterior, dice Fontaine (2015), que "muchos autores no se apartan del análisis secuencial, aunque algunos adopten un enfoque particular y traten de distanciarse de este dogma, sea por el marco analítico de las coaliciones promotoras, las elecciones racionales o el análisis cognitivo" (p. 55).

Los actores en el enfoque cognitivo de politicas públicas. El enfoque cognitivo ${ }^{5}$ de políticas públicas otorga importancia a las ideas, a los valores, a los símbolos, las creencias y el aprendizaje en los procesos de formación de las políticas públicas; es por ello que $\mathrm{Mu}$ ller (2006) habla de enfoque cognitivista. Señala Roth (2006), que "este enfoque estaría caracterizado por su intento de entender las políticas públicas como matrices cognitivas y normativas que conforman sistemas de interpretación o de representación de la realidad y en los cuales los actores públicos y privados insertan sus acciones" (p. 36).

\footnotetext{
4 Los primeros modelos los presenta Roth en su Manual de políticas públicas (Cuervo, 2007a).
}

5 Este enfoque se desarrolló en los años 80 en reacción a las teorías conductistas que manejan como premisa fundamental que las conductas se explican por los intereses individuales, en contraste, los enfoques cognitivistas señalan que algo antecede los intereses, que estos son productos de construcciones sociales, de percepciones $\mathrm{y}$ valores (Fontaine, 2015).
Se estudian a los actores a través del análisis cognitivo de las políticas públicas. El primer modelo es el de la configuración de actores y el referencial de política pública, su autor es Pierre Muller ${ }^{6}$, destacado representante de la escuela francesa de políticas públicas ${ }^{7}$. El segundo modelo, también reconocido como cognitivista, es el Marco de las Coaliciones Promotoras ${ }^{8}$ de Causa (Advocacy Coalition Framework ${ }^{9}$ ). Ambos enfoques cognitivos de políticas públicas han pretendido tomar distancia del modelo secuencial de políticas públicas. Para Gómez (2012), el ACF constituye una alternativa analítica al ciclo de las políticas públicas porque sirve para comprender la totalidad del proceso de dichas políticas.

Es interesante notar que, en esta perspectiva, el problema que plantean las políticas públicas es un poco distinto, pues estos estudios se interesan por las controversias, los conflictos son las consecuencias de las políticas. Aplican en particular a problemas societales y plantean que las políticas públicas son maneras de resolver problemas de sociedad, donde la visión del problema y la relación de poder entre los actores son determinantes. Estos enfoques permiten construir socialmente una situación, definir un problema y proponer una solución (Fontaine, 2015, p. 116).

\footnotetext{
${ }^{6}$ Profesor de ciencias políticas en el Instituto de Estudios Políticos de París. Director de investigación del Centro Nacional de la Investigación Científica de Francia (CNRS) y director del Centro de Estudios e Investigaciones sobre Políticas Públicas de Francia (CEVIPOF) de la Fundación Nacional de Ciencias Políticas de París (Muller, 2006).

${ }^{7} \mathrm{Su}$ libro Les politiques publiques en su edición francesa fue traducido en su versión española por primera vez por los doctores Jean Francois Jolly y Carlos Salazar Vargas, ambos profesores de la Facultad de Ciencias Económicas y Administrativas de la Pontificia Universidad Javeriana de Bogotá, Colombia. Asimismo, el libro Las Políticas Públicas, recibió el apoyo para su edición de la Universidad Externado de Colombia.

8 "El término $<<$ promotora >> traduce el inglés advocacy, un término jurídico para designar la acción de actuar en defensa de alguien o de algo..." (Fontaine, 2015, p. 116).

${ }^{9}$ En adelante se denominará $\mathrm{ACF}$
} 
A continuación, se describen cada uno de los referidos modelos cognitivos:

La configuración de actores y el referencial de política pública. Muller (2000; 2006) agrupa a los actores que pueden incidir en el proceso de decisiones públicas en cuatro círculos de decisión y afirma que, a pesar de que los funcionarios públicos no son todos los actores de las políticas públicas, la administración pública sería: El marco principal de la acción para los participantes en las políticas públicas, sobre todo en Francia. Una dimensión imprescindible del análisis de políticas públicas es la noción de administración pública que tiene múltiples facetas. De un lado, $<<$ da cuerpo a la reivindicación del monopolio de la violencia física legítima>> por parte del Estado y, por esa razón, encarna la dimensión normativa y prescriptiva de las políticas públicas. Del otro, la administración pública toma también la forma de una multitud de organizaciones en cuyo seno unos agentes van a desarrollar estrategias similares a aquellas de los actores privados (...) (Muller, 2006, pp. 72-73).

Debido a lo anterior, Muller (2000; 2006) identifica cuatro círculos de decisión "conformados por un núcleo duro de actores relativamente permanentes y por actores que intervienen de manera más puntual" (Muller, 2006, p. 73), de ahí que se refiera al funcionamiento del medio decisional central propuesto por Catherine Grémion para el caso francés. Se describen a continuación:

El primer círculo es aquel por el cual transitan sin excepción todas las decisiones. Según los países, está compuesto por el Primer Ministro, el Presidente del Consejo, el Canciller (...) Es sobre todo en el seno de este primer círculo de la decisión donde las lógicas de los partidos chocan contra las exigencias de la regulación y que estas últimas vienen consideradas en función de las finalidades políticas.
Al mismo tiempo, está difícilmente asegurada la coherencia de las diferentes políticas públicas sectoriales. El segundo círculo de decisión lo compone el conjunto de las administraciones sectoriales que intervienen cuando su campo está implicado. Buscan sobre todo promover sus intereses sectoriales (y los de los grupos sociales que le están ligados) ajustándolos lo mejor posible a las exigencias globales (...) El tercer círculo de decisión es el de los socios externos al Estado: sindicalistas, gremios, asociaciones, empresas públicas y privadas (...) Los medios de comunicación masivos pueden igualmente jugar un papel importante. Finalmente, el cuarto circulo reúne el conjunto de los órganos políticos (el Parlamento, el Congreso, la Cámara de los Comunes, etc.) y jurisdiccionales (el Consejo Constitucional, el Consejo de Estado y la Corte de Cuentas en Francia, la Corte Suprema en Estados Unidos) que pueden intervenir en la decisión. Su papel puede ser limitado (...) o decisivo (...) (Muller, 2006, pp. 74-75).

Lo anterior es el reflejo de la complejidad, y para ello es fundamental la negociación (en el marco de negociaciones interministeriales) sobre la decisión en materia de las políticas públicas. A juicio de Muller (2006), en el marco de la negociación, son los actores del primer círculo de decisión los que pueden desplegar mejor sus estrategias.

El acceso a los círculos de decisión es un recurso político esencial que le permite a un actor social promover sus intereses (...) Supone la puesta en marcha de una verdadera estrategia de participación en el proceso de fabricación de las políticas públicas (...) (Muller, 2000, p. 119).

El francés Pierre Muller (2006) entiende las políticas públicas como configuraciones de actores y para ello centra su interés en: 1) el problema de la racionalidad de los actores; 2) el papel de la administración pú- 
blica y 3) las redes de actores. En referencia al problema de la racionalidad, Muller plantea la complejidad e incertidumbre de los procesos de toma de decisión y señala que la realidad de los procesos de decisión se encuentra muy distante de los modelos de concepción tradicional ya que los actores no actúan racionalmente y sus preferencias van a evolucionar a lo largo del proceso de formación de la política pública. Sobre el papel de la administración pública ya se explicó a través de los círculos de decisión para el caso francés y, en el tercer punto, se trata de analizar a las políticas públicas como unas redes de actores y así identificar nuevas configuraciones de actores; Muller (2006) lo desarrolla a partir de la noción de redes de políticas públicas (policy network). "No se trata sólo de observar la apertura del medio decisional central a unos actores exteriores, sino de identificar nuevas configuraciones de actores relativamente permanentes que trascienden los clivajes administrativos tradicionales (...)" (Muller, 2006, pp. 80-81).

Lo anterior da cuenta de la estructura cada vez más compleja de los sistemas de decisión pública, donde nos encontramos con una diversificación de los actores que participan en las políticas públicas. Para Muller (2006, p. 84):

(...) La noción de red constituye una ayuda preciosa para entender el papel de los actores en las políticas públicas (...) permite, sobre todo, entender los mecanismos en función de los cuales las diferentes redes van a entrar en contacto y articularse a través de unos procesos de conflicto, de negociación o de coalición. La tarea más importante será identificar los actores susceptibles de actuar en la interfaz entre las diferentes redes, en la medida en que son ellos los que ejercerán la función estratégica de integración de las diferentes dimensiones de la decisión. Llámense policy brokers, empresarios políticos o mediadores (...)
Muller (2006) introduce en su obra la importancia del papel del mediador en el análisis de la política pública. Los mediadores, en palabras del autor, son los actores sociales expertos (skilled social actors), denominados también agentes corredores de políticas públicas (policy brokers), traductores o trascodificadores. Los mediadores se caracterizan, en palabras de Muller (2006, p. 105), por:

$\mathrm{Su}$ capacidad para establecer un puente entre dos espacios de acción y de producción de sentido específicos: lo global y lo sectorial (...) Los mediadores son entonces los agentes que van a encarnar la relación compleja entre las restricciones de lo global y la autonomía de lo sectorial. Por ello, son los mediadores del cambio.

A juicio de este autor, la búsqueda de estos agentes constituye un paso obligado para cualquier análisis de política pública. Los mediadores, como agentes que elaboran el referencial de las políticas públicas (Surel, 2008), realizan los ajustes entre el referencial global y el sectorial. El referencial de la política pública sería la "imagen cognitiva que los actores van a organizar su percepción del sistema, confrontar sus soluciones y definir sus propuestas de acción" (Muller, 2006, p. 98), de allí que "elaborar una política pública se reduce a construir una representación, una imagen de la realidad sobre la cual se quiere intervenir" (Muller, 2006, pp. 97-98).

Este enfoque que hace énfasis en la función cognitiva de la acción pública, ha sido desarrollado por numerosos autores que subrayan el papel de las ideas en el estudio de las políticas públicas. Buscan mostrar que la acción pública se organiza alrededor de marcos que constituyen el universo cognitivo de los actores (...) (Muller, 2006, p. 95).

Un actor, en palabras de Muller (2006, pp. 106-107): 
No podrá construir cualquier sistema de creencia en función de sus necesidades estratégicas, porque las creencias son el resultado de un proceso muy complejo entre el lugar de un grupo en la división del trabajo, y la identidad construida a partir de este lugar (...) el proceso de construcción de un referencial es a la vez la ocasión de una toma de palabra (producción de significación) y de una toma de poder (estructuración de campo de fuerzas) (...) es imposible estudiar la construcción de un referencial sin analizar precisamente las características del grupo que realiza esta operación, sus estrategias y su posicionamiento en el seno del campo del poder (...)

La elaboración de una política pública es a la vez "un proceso por el cual un actor colectivo trabaja sobre su identidad social (...)" (Muller, 2006, p. 106). "Alrededor de una política pública existe la constitución de un sistema de actores jerarquizado, donde los mediadores tienen la parte más influyente en razón de su apego común al referencial" (Surel, 2008, p. 43).

Finalmente, Muller (2006) señala que el proceso de imposición/aceptación del cambio de referencial pasa por el funcionamiento diferenciado de varias instancias a las cuales denomina foros, que son escenarios específicos de construcción de la realidad social; en cada uno de estos foros existe una variedad de actores, como se aprecia en la tabla 3.
Esta distinción entre los diferentes foros deja ver con claridad la importancia y complejidad del papel que tienen los actores (grupos de interés, redes, comunidades de políticas) frente al Estado para explicar por qué ciertos temas entran en la agenda de gobierno y otros no (Muller, 2006; Fontaine, 2015).

Los actores en el Marco de las Coaliciones de Causa (Advocacy Coalition Framework). El $\mathrm{ACF}^{10}$, a juicio de Gómez (2012), "constituye una alternativa analítica al ciclo de las políticas públicas porque sirve para comprender la totalidad del proceso de dichas políticas. Este enfoque delimita la política pública a partir del subsistema y la define como sistema de creencias" (p. 27). Parte de un enfoque endógeno, es decir, cómo unos actores estatales y no estatales se unen en la acción pública; existe un núcleo alrededor del cual se van aglutinando los actores y es a través de esta aglutinación que se van conformando las coaliciones promotoras. Las coaliciones se forman de las creencias de sus miembros (Fontaine, 2015).

\footnotetext{
10 “(...) rompe intencionalmente con una visión jerárquica (top-down), de las políticas públicas y trata de evidenciar que el proceso viene de abajo hacia arriba (bottom-up)" (Sabatier, citado por Fontaine, 2015, p. 117), es decir, desde los actores no-estatales hacia el Estado (Fontaine, 2015).
}

Tabla 3

Los foros y el papel de los actores.

\begin{tabular}{ll}
\hline \multicolumn{1}{c}{ Foros } & \multicolumn{1}{c}{ Actores } \\
\hline $\begin{array}{l}\text { El foro científico de } \\
\text { los economistas }\end{array}$ & $\begin{array}{l}\text { Los economistas buscan la excelencia académica en el paradigma dominante y la } \\
\text { innovación intelectual. }\end{array}$ \\
$\begin{array}{l}\text { El foro de la } \\
\text { comunicación política }\end{array}$ & $\begin{array}{l}\text { Los actores políticos tienen la voluntad de participar en la construcción de } \\
\text { coaliciones de los partidos políticos susceptibles de acceder al poder. }\end{array}$ \\
$\begin{array}{l}\text { El foro de las } \\
\text { comunidades de } \\
\text { políticas públicas }\end{array}$ & $\begin{array}{l}\text { Lugar donde se fabrican las recetas por las comunidades de políticas públicas. } \\
\text { políticas públicas. }\end{array}$ \\
\hline
\end{tabular}

Fuente: elaboración propia con base en Muller (2006). 
Se destaca la consideración de las ideas en el proceso de formación de las políticas públicas, de manera similar al modelo de Muller (2006), por lo que ambos modelos se destacan por incorporar los aspectos cognoscitivos ${ }^{11}$. En este último, "los diferentes actores se agrupan en coaliciones que intentan trasladar sus sistemas de creencias a las políticas públicas y, para ello, participan en procesos continuos de aprendizaje" (Martinón, 2007, p. 281).

El marco de las coaliciones promotoras, desarrollado por Sabatier ${ }^{12}$ y Jenkins-Smith, ${ }^{13}$ se enfoca en la interacción de coaliciones promotoras (cada una compuesta por actores de diversas instituciones que comparten un conjunto de creencias sobre políticas) dentro de un subsistema de políticas. El cambio de políticas depende tanto de la competencia dentro del subsistema como de los eventos fuera del subsistema. El marco dedica mucho tiempo a delinear los sistemas de creencias de las elites políticas y a analizar las condiciones en las cuales puede darse el aprendizaje orientado a las políticas entre coaliciones (Sabatier, 2010, p. 12).

Este marco conceptual para estudiar el proceso de las políticas públicas, y que se remonta a los años 1981-1982, ha sido desarrollado por los referidos autores para resolver problemas que involucran grandes conflictos de objetivos, así como también, importantes discusiones técnicas y muchos actores de diferentes niveles de gobierno (Hoppe y Peterse, citado por Sabatier y Weible, 2010).

\footnotetext{
11 Considera el papel de los valores, los símbolos, las creencias, el conocimiento y el aprendizaje en los cambios que se producen en las políticas públicas.

12 Paúl A. Sabatier es profesor de Ciencias Ambientales y Política de la Universidad de California.

${ }^{13}$ Hank C. Jenkins-Smith es profesor de Ciencia Política y director asociado del Centro de Investigación Social Aplicada.
}

Entre las premisas ${ }^{14}$ de este modelo se encuentran en referencia a los actores, las siguientes: 1) la unidad de análisis no se limita a la estructura gubernamental, sino a un subsistema de política que está compuesto por una variedad de actores tanto públicos como privados, que están implicados o interesados en un problema de política o en una controversia y, 2) se incluyen como actores al interior del subsistema a los periodistas, investigadores y a los analistas de políticas, dado la importancia de su rol en la difusión de ideas y otros actores de diferentes niveles de gobierno activos en el proceso de formulación e implementación de las políticas públicas (Roth, 2007; Roth, 2008).

Señalan Sabatier y Weible (2010) que el marco de coaliciones de causa no solamente incluye en la formulación de las políticas "al tradicional <<triángulo de hierro>> conformado por legisladores, funcionarios de organismos y líderes de grupos de interés, sino también a los investigadores y periodistas que se especializan en esa área de políticas" (Heclo y Kingdon, citados por Sabatier y Weible, 2010, p. 206). Asimismo, estos autores incorporan a los funcionarios judiciales, quienes también intervienen en los subsistemas de políticas (Sabatier y Weible, 2010).

En este modelo se destaca la participación de los investigadores (científicos universitarios, analistas de políticas, consultores, etc.), como uno de los actores más importantes, ya que la información científica y técnica, en palabras de Sabatier y Weible (2010), juega un rol fundamental en la modificación de las creencias.

\footnotetext{
${ }^{14}$ Otra premisa importante del modelo es considerar. en los cambios de políticas, una perspectiva temporal de larga duración (diez años o más), también el marco de las coaliciones promotoras asume que las creencias de los participantes son muy estables, lo que dificulta los cambios importantes de políticas (Sabatier y Weible, 2010).
} 
Sabatier y Jenkins-Smith, sin desestimar los factores externos ${ }^{15}$ de la política pública, le otorgan especial importancia en su modelo a los factores internos, específicamente por los efectos causados por el aprendizaje político que provocan cambios en las políticas públicas.

El aprendizaje político es el efecto producido sobre los actores y sobre la misma política por la suerte de retroacción interna a la política que se produce con el paso del tiempo. La experiencia cotidiana que adquieren los actores de la política se traduce en una adaptación o modificación de los comportamientos y actitudes, en una adquisición de un saber práctico. El aprendizaje hace referencia a los aspectos cognitivos que resultan de la práctica de las políticas públicas. Según Sabatier y Jenkins-Smith, este aprendizaje político es también un factor que influye sobre los contenidos y cambios en las políticas públicas en el transcurso de los años (...) (Roth, 2006, pp. 168-169).

El marco de las coaliciones promotoras sostiene que los participantes buscarán aliarse con personas que tengan las mismas creencias sobre las políticas entre los actores mencionados anteriormente de varios niveles de gobierno. La coordinación incluye algún grado de trabajo conjunto para alcanzar objetivos de políticas similares. Las coaliciones promotoras proveen la herramienta más útil para aunar el comportamiento de cientos de organizaciones e individuos involucrados en un subsistema de políticas que puede incluir de dos a cinco coaliciones promotoras (Sabatier y Weible, 2010).

\footnotetext{
${ }^{15}$ Son el desarrollo socioeconómico, la política (elecciones, partidos), los gremios y los altos funcionarios de la administración pública (Hecho; Rose, citados por Roth, 2006).
}

Los cambios de política pública resultarían del trabajo y la lucha de estas coaliciones de actores. A partir de los recursos que encuentran tanto en el entorno pertinente al subsistema de la política -los factores externos-, como en su interior -el aprendizaje político $^{16}$-, las coaliciones intentarían hacer prevalecer su punto de vista, sus valores e intereses para lograr su dominio en la conducción de la política pública (Roth, 2006, p. 170).

En este modelo es muy importante el dominio experto $^{17}$ que ejercen algunos actores.

El rol de los expertos ${ }^{18}$ (sean académicos, tecnócratas o profesionales contratados para una actividad puntual) es central porque es lo que necesitan las coaliciones para aprender con el fin de incidir en la política. Precisamente porque las coaliciones promotoras se organizan a partir del conocimiento, de la comprensión (que puede ser técnica) de un problema, el aprendizaje recoge una importancia nueva, es parte constitutiva del proceso político. Por ejemplo, alude a cómo se forman o se capacitan actores entre los profesionales de la política (Fontaine, 2015, p. 118).

\footnotetext{
16 "Existen dos niveles de aprendizaje: uno técnico, que consiste en sacar lecciones de experimentos del pasado, y uno político, que es una experticia que permite a ciertos actores conseguir una postura privilegiada en un conflicto o en una coalición, jugar un rol clave en un momento dado (...) El aprendizaje es parte del proceso político e incide en la asignación de valores (...) el aprendizaje dentro del sistema de creencias de una coalición varía en función del nivel de conflicto, del posible seguimiento al tema y de la existencia de un foro profesionalizado" (Fontaine, 2015, p. 118).

${ }^{17}$ Lo que explica, a juicio de Fontaine (2015), que el monetarismo y el neoliberalismo se impusieron en un momento clave. "Las ideas se imponen, no solo porque son buenas, sino porque es el buen momento" (p. 116).

18 "Hay dos categorías de expertos: los que se dedican al trabajo con el Estado, cuyo aprendizaje responde a la vocación weberiana del servidor público, y los actores de la sociedad civil que se involucran en la incidencia política, para quien existen cada vez más carreras de altos estudios, incluso en las universidades donde se forman aquellos funcionarios públicos" (Fontaine, 2015, p. 118).
} 
En la práctica aparece otro actor importante que Sabatier denomina mediadores de política (policy brokers), similar a la figura de los mediadores que nos presenta Muller (2006). Este tipo de actor, "que no pertenece claramente a una u otra de las coaliciones, juegan un papel de intermediarios o de puente entre las concepciones que se enfrentan e intentan encontrar una solución $<<$ razonable $>>$ o $>>$ practicable $>>$ al problema” (Roth, 2006, p. 171).

Las coaliciones se definen a partir de la comprensión de un problema -lo cual nos remite nuevamente a la elaboración de agenda- y definen una línea base a partir de qué quieren los actores o qué creen ellos que hay que resolver; luego, cómo piensan que se puede resolver este problema. De una vez, se da el paso de la definición de un problema a la formulación de una solución, a partir de la cual opera esta alianza (...) Es a priori una coalición de un número bastante restringido de individuos que, poco a poco, inscriben a la agenda de política un tema alrededor del cual se van a aglutinar otras fuerzas sociales y políticas, que lo convierten en un eje estructurador de las políticas (Fontaine, 2015, p. 118).

Finalmente, el ACF se presenta en la actualidad, en palabras de Bergeron et al, (citado por Fontaine 2015), "como uno de los métodos más sofisticados de análisis del rol de los actores no-estatales en la acción pública" (p. 152).

Las primeras investigaciones aplicando el ACF versaron sobre la política de energía y la política ambiental de los Estados Unidos, campo de investigación de Sabatier y Jenkins-Smith. Siendo en Europa y en Estados Unidos, donde más se ha aplicado el ACF, también ha sido aplicado a temas de políticas públicas (temas económicos y sociales) en países tales como: África, Asia, América del Sur, Australia y Canadá (Sabatier y Weible, 2010).
Los actores en el modelo decisional de Dente y Subirats. Dente y Subirats (2014) plantean las decisiones públicas en entornos plurales y en sistemas democráticos, considerando la complejidad, la pluralidad de visiones, la heterogeneidad de intereses, el debate y la deliberación pública para la búsqueda de soluciones posibles. Estos autores pretenden ir más allá de las soluciones incrementales y quieren transformar el entorno con sus herramientas.

La capacidad de tomar una decisión no incremental en la solución de un problema de políticas complejo es más probable en la medida en que el proceso decisional muestra un nivel de complejidad similar, al menos en lo relativo al número y a la diversidad de los actores directa o indirectamente implicados, a la cantidad y el tipo de intereses afectados por el problema o la solución (Dente, citado por Dente y Subirats, 2014, p. 269).

Dentro de este marco, los autores diseñan un modelo realista del proceso de toma de decisiones con el propósito de lograr una transformación, intencional y no incremental, de una política pública, es decir, de cómo responder a un problema colectivo, por lo que sugieren ir más allá de Charles Lindblom.

Podríamos resumir a continuación su planteamiento central:

Los resultados de un proceso de toma de decisiones de política pública dependen de la interacción de tipos diferentes de actores, con diferentes objetivos y roles diferentes que, dentro de una network o entramado que puede tener diferentes características, se intercambian recursos, utilizando diferentes modalidades de interacción, para conseguir un acuerdo, dentro de un contexto decisional determinado (Dente y Subirats, 2014, p. 70). ${ }^{19}$

\footnotetext{
${ }^{19}$ Negritas de los autores
} 
Tabla 4

Elementos del modelo decisional para el análisis de las decisiones de políticas públicas.

\begin{tabular}{|c|c|}
\hline Elementos & Clasificación \\
\hline Identificar a los actores & Son aquéllos que llevan a cabo las acciones relevantes, los que actúan \\
\hline Objetivos de los actores & a) Objetivos de contenido y b) objetivos de proceso \\
\hline Recursos de los actores & $\begin{array}{l}\text { a) Recursos políticos; b) recursos económicos; } \\
\text { c) recursos legales; y d) recursos cognitivos }\end{array}$ \\
\hline Tipos de actores & $\begin{array}{l}\text { a) Actores políticos; b) actores burocráticos; } \\
\text { c) actores que representan intereses especiales; } \\
\text { d) actores que representan intereses generales } \\
\text { y e) los expertos }\end{array}$ \\
\hline $\begin{array}{l}\text { Roles de los actores en la } \\
\text { interacción }\end{array}$ & $\begin{array}{l}\text { a) El de promotor o iniciador; b) el de director; } \\
\text { c) el de opositor; d) el de aliado; } \\
\text { e) el de mediador; f) el de gatekeeper (portero); } \\
\text { y g) el de filtro }\end{array}$ \\
\hline $\begin{array}{l}\text { La forma de las networks } \\
\text { decisionales }\end{array}$ & Según el grado de complejidad en que los actores interactúan \\
\hline $\begin{array}{l}\text { La complejidad de } \\
\text { un proceso decisional y de una } \\
\text { network }\end{array}$ & Se puede medir analizando la red de los actores involucrados \\
\hline El contenido de la decisión & $\begin{array}{l}\text { Sería el factor capaz de predecir cuáles serán los actores } \\
\text { más influyentes en la adopción de la opción final }\end{array}$ \\
\hline $\begin{array}{l}\text { Las modalidades de } \\
\text { interacción entre los actores }\end{array}$ & $\begin{array}{l}\text { a) Confrontación; b) negociación y c) colaboración. } \\
\text { Estas modalidades de interacción entre actores } \\
\text { se deben relacionar con las arenas decisionales: } \\
\text { a) arenas técnicas; b) arenas políticas; } \\
\text { c) arenas sociales; y d) arenas institucionales }\end{array}$ \\
\hline $\begin{array}{l}\text { El contexto } \\
\text { de la decisión }\end{array}$ & $\begin{array}{l}\text { El contexto o entorno decisional es el conjunto de factores y condiciones, } \\
\text { estructurales o coyunturales, que influyen en los procesos decisionales } \\
\text { y contribuyen a determinar los resultados. Se mide en términos de: } \\
\text { a) estabilidad; b) cambio turbulento y c) cambio previsible }\end{array}$ \\
\hline
\end{tabular}

Fuente: elaboración propia con base en Dente y Subirats (2014).

En la tabla anterior se muestran los elementos del modelo decisional y su clasificación, para el análisis de las decisiones de políticas públicas.

\section{¿Dónde están los ciudadanos en el enfoque de análisis de políticas públicas?}

En el estudio de la política pública ha sido predominante el enfoque del ciclo de la política (Roth, 2016; Aguilar, 2017), que entiende la política como el resultado de un proceso de varias operaciones (la formación de la agenda de gobierno, la defi- nición del problema, la elaboración de las opciones para su solución, la decisión, la implementación y la evaluación), consideradas necesarias para que la política sea efectiva. Posteriormente, los estudios de políticas públicas introducen los enfoques cognitivos desde los años ochenta, destacando la importancia de las ideas y de las creencias, no obstante, en estos modelos y enfoques, el ciudadano no es considerado un actor importante.

Esto se debe fundamentalmente a dos razones, según Aguilar (2017, p. 27): 
Una es político-institucional y la otra es disciplinaria. La primera razón señala que la omisión se debe al estilo de gobernar que fue el dominante durante la segunda mitad del siglo XX y que se caracterizó por la amplia autonomía del gobierno en sus decisiones directivas. La segunda razón, de naturaleza disciplinaria, tiene que ver con el modo como fue conceptualizado el objeto de conocimiento de la disciplina, la política pública, que fue entendida básicamente como decisión, como el proceso y el resultado de una decisión de gobierno (...)

En el plano de la realidad, en referencia a la participación ciudadana, explica Aguilar (2017) que:

(...) La política pública nació y se ha desarrollado en el marco de un proceso de gobierno con alta autonomía gubernamental, en correspondencia con un contexto de baja participación ciudadana, en el que la ciudadanía no exigía transparencia ni rendición de cuentas, a pesar de las fallas institucionales y técnicas que eran evidentes en las políticas económicas, sociales, de infraestructura, que decidía el agente gubernamental (...) La mayor parte de los ciudadanos no demandaba participar en la definición de la agenda y en la elaboración de las políticas y tampoco se movilizaba para exigir transparencia y reclamar al poder ejecutivo que rindiera cuentas de sus decisiones, actos y empleo de los recursos públicos. El proceso de gobernar se asentaba más bien en la relación que tenía lugar entre los poderes ejecutivo y legislativo, sea en régimen parlamentario o presidencial, más que en una relación entre ellos y los ciudadanos (...) En mucho esta actitud se debió a que la ciudadanía estaba relativamente satisfecha en los primeros años de la posguerra con sus gobiernos que fomentaban la reconstrucción y desarrollo industrial de sus países, hacían crecer el empleo, modernizaban la vida, extendían los niveles de bienestar y seguridad social (Aguilar, 2017, pp. 27-28).
En ese contexto, el gobierno sustentaba sus decisiones de política en el análisis y cálculo de un círculo de expertos probados, conocedores del problema público, sin embargo, las fallas y limitaciones (institucionales, financieras, administrativas, políticas) de las políticas públicas, junto con los escándalos de corrupción y abusos, han tenido como efecto que la ciudadanía reclame participar en las deliberaciones y decisiones públicas (Aguilar, 2017).

En el plano del conocimiento, si bien es cierto que el enfoque de análisis de políticas desestimó el tema de la comunicación, dice Aguilar (2017), que "el autor que abre la puerta a la consideración teórica de la necesidad e importancia de la comunicación de la política es Giandomenico Majone, con su libro Evidencia, argumentación y persuasión en la formulación de políticas ${ }^{20 "}$ (p. 29).

La política pública según Majone, no es sólo una decisión, centrada en la razón técnica, la política ante el público ciudadano (con opositores y aliados, conocedores y desinformados) implica razón dialógica, interlocución dentro y fuera del gobierno con múltiples actores, que rebasa y complementa la razón técnica y exige conversación y debate entre la autoridad pública y el público ciudadano (Aguilar, 2017).

Majone habló de "razón dialéctica», no de comunicación. Pero su contribución a la disciplina es haber dejado en claro que el análisis, diseño y desarrollo de la política pública en un régimen democrático implica interlocución, debate, proceso de argumentación y no sólo técnicas formales de solución de problemas y de costo-eficiencia, llevadas a cabo por una élite profesional de alta competencia técnica, pero que no considera necesario ni se siente obligada a dialogar con el público ciudadano para comunicarles y justificar la política pública que han diseñado y convencerlo acerca de su idoneidad (Aguilar, 2017, p. 30).

${ }^{20}$ Cursivas del autor 
Estamos de acuerdo con Aguilar (2017, p. 33) cuando afirma que:

La comunicación del gobierno con la sociedad comienza a tener lugar antes de elaborar la política y durante su elaboración, y que la comunicación se desarrolla con un mayor o menor número de sectores según la naturaleza del asunto público y según el grado de interés e involucramiento de los ciudadanos en el asunto. La comunicación ya no ocurre únicamente después de la política decidida sino antes del proceso de decisión y a lo largo del proceso.

Finalmente, dentro del enfoque de políticas encontramos definiciones del concepto de política pública, que, a nuestro juicio, incorporan al ciudadano como un actor fundamental. La primera definición, es de Velásquez (2009), quien luego de estudiar 29 definiciones, las catalogó de incompletas y propone:

Política pública es un proceso integrador de decisiones, acciones, inacciones, acuerdos e instrumentos, adelantado por autoridades públicas con la participación eventual de los particulares, y encaminado a solucionar o prevenir una situación definida como problemática. La política pública hace parte de un ambiente determinado del cual se nutre y al cual pretende modificar o mantener (p. 156).

En la segunda definición, (Cuervo, 2007b), realiza una revisión crítica del término y, entiende la política pública como:

Las actuaciones de los gobiernos y de otras agencias del Estado, cuando las competencias constitucionales así lo determinen - en desarrollo de ese marco y de las demandas ciudadanas- caracterizadas porque constituyen flujos de decisión -o una decisión específica- orientadas a resolver un problema que se ha constituido como público, que moviliza recursos institucionales y ciudadanos bajo una forma de representación de la sociedad que potencia o delimita esa intervención (p. 82) ${ }^{21}$.

${ }^{21}$ Cursivas del autor.

\section{Conclusiones}

Un aporte importante del enfoque de análisis de políticas es que identifica a los actores que intervienen en las decisiones públicas. Se destaca en el enfoque cognitivo la importancia que se le da a los mediadores, como agentes que elaboran el referencial de las políticas públicas, y en el modelo de las coaliciones de causa, la importancia que se le otorga también al papel del mediador y a los expertos.

En este sentido, la información científica y técnica juega un rol fundamental en la modificación de las creencias que provocan cambios en las políticas públicas a favor de un referencial: en este caso es el mercado. En efecto, los enfoques y modelos no son neutrales, siempre obedecen a un paradigma que se pretende imponer.

Dente y Subirats (2014), en un modelo decisional más realista, identifican como actores, además de los expertos, los actores políticos, los actores burocráticos, los actores que representan intereses especiales y actores que representan intereses generales agrupados en networks decisionales. Es uno de los modelos más completos para estudiar a los actores dentro del enfoque de análisis de políticas; no solo considera los tipos de actores, sino también se interesa por sus objetivos, recursos, roles en la interacción, forma de las networks decisionales de acuerdo con su complejidad en que los actores actúan, el contenido de la decisión, las modalidades de interacción entre los actores, y le dan importancia al contexto y su influencia en los procesos decisionales.

Los modelos teóricos estudiados presentan aportes al estudiar a los actores dentro del proceso de formación de la política pública, con énfasis en algunos actores, de interés para cada modelo. Sin embargo, los investigadores del enfoque de análisis de políticas deben realizar un esfuerzo teórico en el estudio del ciudadano como un actor fundamen- 
tal a lo largo del proceso de formación de la política pública. Si las políticas públicas son flujos de decisiones y de acción de los gobiernos, orientadas a resolver problemas sociales, el ciudadano debe estar presente tanto en los modelos y enfoques (en el conocimiento), como en la realidad, participando en la toma de las decisiones públicas.

\section{Referencias}

Aguilar, L. F. (2017, abril). Entrevista sobre Comunicación Política. Más Poder Local Magazine. (31), 26-33. Recuperado de http://www.maspoderlocal.es/ediciones/comunicacionpoliticas-publicas-ambito-local-n31/

Balestrini, M. (1998). Estudios documentales, teóricos, análisis de discurso y las historias de vida. Una propuesta metodológica para la elaboración de sus proyectos. Caracas. Venezuela: BL Consultores Asociados.

Cuervo, J. I. (2007a). La definición del problema y la elaboración de la agenda. En Ensayos sobre Políticas Públicas, pp. 151-165. Universidad Externado de Colombia, Bogotá, D.C., Colombia.

Cuervo, J. I. (2007b). Las políticas públicas: entre los modelos teóricos y la práctica gubernamental (una revisión a los presupuestos teóricos de las políticas públicas en función de su aplicación a la gestión pública colombiana. En Ensayos sobre Políticas Públicas, pp. 67-95. Universidad Externado de Colombia, Bogotá, D.C., Colombia.

Dente, B. y Subirats, J. (2014). Decisiones Públicas. Análisis y estudio de los procesos de decisión en politicas públicas. Barcelonaa: Ariel.
Fontaine, G. (2015). El análisis de politicas públicas. Conceptos, teorías y métodos. Barcelona: Anthropos.

Gómez, M. I. (2012). El Marco de las Coaliciones de Causa -Advocacy Coalition Framework. Revista Ópera. (12), 11-30. Recuperado de http:// revistas.uexternado.edu.co/index. php/opera/article/view/3648/3740

Martinón, R. (2007). La incorporación de las ideas al análisis de políticas públicas en el marco de las coaliciones promotoras. Gestión y Política Pública, 16(2), 281-318. Recuperado de http://www.gestionypoliticapublica.cide.edu/num_anteriores/Vol. XVI_No.II_2dosem/Ruth_Martinon_Quintero.pdf

Muller, P. (2000). Los círculos de decisión en las políticas públicas. Innovar, 10(15), 111-120. Recuperado de http://www.revistas.unal.edu.co/index.php/innovar/article/view/24212

Muller, P. (2006). Las políticas públicas. Bogotá, D.C.: Universidad Externado de Colombia.

Roth, A. N. (2006). Políticas Públicas. Formulación, implementación y evaluación. Bogotá, D.C.: Aurora.

Roth, A. N. (2007). Enfoques y teorías para el análisis de las políticas públicas, cambio de la acción pública y transformaciones del Estado. En, Ensayos sobre Políticas Públicas, (29-63). Universidad Externado de Colombia, Bogotá, D.C., Colombia.

Roth, A. N. (2008). Perspectivas para el análisis de las políticas públicas: ¿de la razón científica al arte retórico? $R e$ vista Estudios Políticos, (33), 67-91. Recuperado de http://www.redalyc. org/articulo.oa?id=16429061004 
Roth, A. N. (2016). La enseñanza del análisis de políticas públicas en los programas universitarios de Ciencia Política en Colombia. Revista Estudios Políticos, (49), 260-283. Recuperado de http://aprendeenlinea.udea.edu. co/revistas/index.php/estudiospoliticos/article/view/25973/20781239

Sabatier, P. A. (2010). Se necesitan mejores teorías. En, P. Sabatier (Ed.), Teorías del proceso de las políticas públicas, (5-19). Buenos Aires: Westview Press. Recuperado de file://C:/Users/USUARIO/Downloads/920253696.SABATIER-\%20 Se\%20necesitan\%20mejores\%20 teor\%C3\%ADas\%20(2).pdf

Sabatier, P. A. y Weible, Ch. M. (2010). El marco de las coaliciones promotoras. En, P. Sabatier (Ed.), Teorías del proceso de las políticas públicas, (203-238). Buenos Aires: Westview Press. Recuperado de http:// maxicamposrios.com.ar/wp-content/ uploads/2014/03/Sabatier-P.-WeibleC.-El-marco-de-las-coaliciones-promotoras.-Innovaciones-y-clarificaciones.pdf
Santibáñez, A., Barra, M. y Ortiz, P. (2005). La racionalidad de los actores en políticas públicas: un esquema teórico para entender el funcionamiento de las democracias modernas. Recuperado de http://www.colpos.mx/tabasco/ diplomado/3y4demarzo/actores_en_ politica.pdf.

Subirats, J. (2015). Prólogo del libro El Análisis de Políticas Públicas. Conceptos, teorías y métodos, (XI-XIII). Barcelona: Anthropos.

Surel, Y. (2008). Las políticas públicas como paradigmas. Revista Estudios Políticos, (33), 41-65. Recuperado de http://aprendeenlinea.udea.edu. co/revistas/index.php/estudiospoliticos/article/view/1942/5845

Velásquez, R. (2009). Hacia una nueva definición del concepto política pública. Revista Desafios, 20(1), 149187. Recuperado de https://revistas. urosario.edu.co/index.php/desafios/ article/view/433/377 